\title{
SEMIGROUP COMPACTIFICATIONS BY GENERALIZED DISTAL FUNCTIONS AND A FIXED POINT THEOREM
}

\author{
R.D. PANDIAN \\ Department of Mathematics \\ North Central College \\ Naperville, IL 60566 \\ (Received August 15, 1989)
}

The notion of "Semigroup compactification" which is in a sense, a generalization of the classical Bohr (almost periodic) compactification of the usual additive reals $R$, has been studied by J.F. Berglund et. al. [2]. Their approach to the theory of semigroup compactification is based on the Gelfand-Naimark theory of commutative $C^{*}-$ algebras, where the spectra of admissible $C^{*}$-algebras, are the semigroup compactifications. H.D. Junghenn's extensive study of distal functions is from the point of view of semigroup compactifications [5]. In this paper, extending Junghenn's work, we generalize the notion of distal flows and distal functions on an arbitrary semitopological semigroup $S$, and show that these function spaces are admissible $C^{*}-$ subalgebras of $\mathrm{C}(\mathrm{S})$. We then characterize their spectra (semigroup compactifications) in terms of the universal mapping properties these compactifications enjoy. In our work, as it is in Junghenn's, the Elils semigroup plays an important role. Also, relating the existence of left invariant means on these algebras to the existence of fixed points of certain affine flows, we prove the related fixed point theorem.

\section{PRELIMINARIES.}

Let $\mathrm{S}$ be a semitopological semigroup (binary operation separately continuous) with a Hausdorff topology, and $C(S)$ denote the $C^{\star}$-algebra of a11 bounded complex valued continuous functions on $S$ (all topologies are assumed to be Hausdorff). For $s \in S$, define $\mathrm{L}_{S}$ and $R_{s}$ on $C(S)$ byL $L_{S} f(t)=f(s t)$ and $R_{s} f(t)=$ $f(t s)$ ( $f \in C(S)$ and $t \varepsilon S$ ). A subspace $F$ of $C(S)$ is left (right) translation invariant if $L_{s} F \subseteq F\left(R_{S} F \subseteq F\right)$. It is translation invariant if it is both left and right translation invariant. A $C^{*}$-subalgebra $F$ of $C(S)$ is called admissible if it is translation invariant, contains the constant functions, and is left-m-introverted, 1.e., $\left.T_{x} f(\cdot)=x\left(L_{(}\right) f\right)$ is a member of $F$ whenever $f \in F$ and $x$ belongs to the spectrum of $F$ (the space of al1 nonzero continuous homorphisms on $F$ ). In this case, $T_{x}: F \rightarrow F$ is called the left-m-introversion operator determined by $x$. A right topological compactification of $S$ is a pair $(X, \alpha)$, where $X$ is a compact right topological semigroup (1.e., $X$ is a compact semigroup with the mapping $x+x y: X+X$ continuous for al1 y $\varepsilon X)$, and $\alpha: S+X$ is a continuous homorphism with dense image such that for each $s \in S$, the mapping $x+\alpha(s) x: X \rightarrow X$ is continuous. If, in addition, $\alpha^{*} C(X)=F$ where $F$ is an admissible subalgebra of $C(S)$ and $\alpha^{*}: C(X)+C(S)$ is the dual mapping $f+f o \alpha$, then $(X, \alpha)$ is called an F-compactification of $S$. A right topological compactification $(X, \alpha)$ of $S$ is said to be maximal with respect to a 
property $P$ if $(X, \alpha)$ has the property $P$, and whenever $(Y, \beta)$ is a right topological compactification of $S$ with the property $P$, then there exisis a continuous homumorphisin $\nu: X+Y$ such that $v_{0} \beta=\alpha$. The factorization of the napping $\beta$ by $\alpha$ is referred to as a universal mapping property of $(X, x)$. F-compactifications are maximal with respect to the property that $\alpha^{\star} C(X) \subseteq F[2$, [I[ Theorem 2.4]. This result will be used frequently without specific reference to it. For a fixed admissible subalgebra $F$ of $C(S)$, all F-compactifications of $S$ are algebraically and topologically isomorphic, and hence, we speak of the F-compactification of $S$. If $F$ is a norm closed, conjugate closed subspace of $\mathrm{C}(\mathrm{S})$ containing constants, then a $\mu \in F^{*}$ (dual of $F$ ) is called a mean on $F$ if $\mu(1)=1=\|\mu\|$. If $F$ is further closed under multiplication (pointwise), a mean $\mu$ on $F$ is called multiplicative if $\mu(f g)=$ $\mu(f) \mu(g), f, g \in F$. We denote the set of all means [multiplicative means] on $F$ by $M(F)[M M(F)]$. With $w^{*}$-topology, $M M(F)$ is compact and it is the $w^{*}-c l o s u r e$ of $e(S)$, where $e$ is the evaluation $\operatorname{map}\{e(s)(f)=f(s)\}$. We note that (MM(F), e) is an Fcompactification of $S$, and we call this the canonical F-compactification of $S$. We will need the admissible subalgebra $L M C(S)=\left\{f \in C(S): s \rightarrow \mu\left(L_{s} f\right)\right.$ is continuous for all' $\mu \in M M C(S)$ \} in the sequel. We note that the LMC(S)-compactification is maximal with respect to the property that it is a right topological compactification of $S$ [2, II I Theorem 4.5].

A flow is a triple $(S, X, \pi)$, where $S$ is a semitopological semigroup, $X$ is a compact topological space, and $\pi: S \rightarrow X^{X}$ is a continuous homomorphism such that $\pi(s): X+X$ is continuous for each $s \in S$. We often write $(S, X)$ for $(S, X, \pi)$ and sx for $\pi(s) x$. $\quad X^{X}$ is a compact right topological semigroup (with respect to the product topology and function composition) of all self maps of $X$. We denote the Ellis semigroup, the clsoure of $\pi(S)$ in $X X$, by $E(S, X)$. $E(S, X)$ is then a compact right topological semigroup. If $X$ is a convex subset of a real or a complex vector space, and $\pi(s): X+X$ is affine for each $s$ in $S$, then $(S, X)$ is called an affine flow. A point $X$ in $X$ is called a fixed point of the flow $(S, X)$ if $s x=X$ for each $s$ in $S$. If $Y$ is a closed invariant subspace of $X$, then $(S, Y)$ is a flow under the restricted action. A flow $(S, X, \pi)$ is called distal if, whenever $x, y \varepsilon X$ such that $1 i m s_{1} x=1 i m s_{1} y$ for some net $\left(s_{i}\right)$ in $S$, then $x=y$. Let $f \varepsilon L M C(S)$ and $z$ be the closure of $R_{S} f$ in the

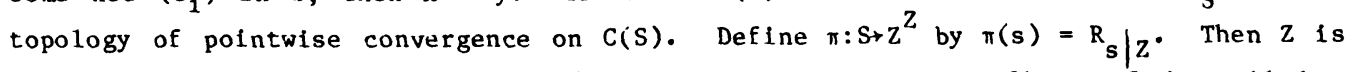
pointwise compact [6], and $(S, Z, \pi)$ is easily seen to be a flow. $f$ is called a distal function if the flow (S, Z, $\pi$ ) is distal. H. D. Junghenn has shown that D(S), the set of all distal functions, is an admissible subalgebra of $C(S)$ and that a function $f \in \operatorname{LMC}(S)$ is distal iff $u e v(f)=u v(f)$ for $u, v$ in $X$ and $e \varepsilon E(X)$, the idempotents of $X$, where $(X, \alpha)$ is the LMC $(S)$-compactification of $S$. Also, he has proved that the $D(S)$-compactification $(Y, B)$ is maximal with respect to the property that $x e y=x y$ for all $x, y$ in $Y$, e $\varepsilon E(Y)$ [5, Theorem 3.4].

\section{GENERAL,IZED DISTAL FUNCTIONS.}

Let $(S, X, \pi)$ be a flow and $E(S, X)$, the Ellis semigroup. Define $E(S, X)^{n}=\left\{g_{1} g_{2} \ldots \ldots \ldots g_{n} \mid g_{1} \varepsilon E(S, X)\right\}$. Then $\overline{E(S, X)^{n}}$ and $n=(S, X)^{n}$ are both compact right topological semigroups. We note that $E(S, X)^{n}$ is nonempty as compact right topological semigroups have idempotent elements [4]. 
DEFINITION 1. A flow (S, $X, \pi)$ is $n$-distal ( $\xi(x)=\xi(y)$ for some $\xi \varepsilon E(S, x)$, then $\zeta(x)=\zeta(y)$ for every $\zeta \varepsilon E(S, x)^{n}$ $\left(\zeta \in \cap E(S, X)^{n}\right)$.

DEFINITION 2. A function $f \in L M C(S)$ is said to be $n$-distal (o-distal), if the flow ( $S, Z, \pi)$, where $Z$ is the closure of $R_{S} f$ in the topology of pointwise convergence

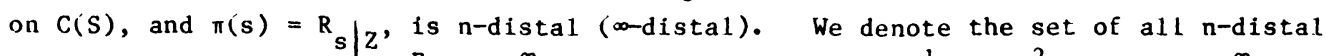

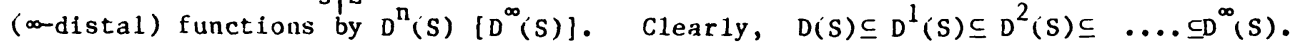

PROPOSITION 3. A flow $(S, X, \pi)$ is $n$-distal if and only if, whenever $x, y \varepsilon X$ such that $\lim s_{i} x=1 i m s_{i} y$ for some net $\left(s_{i}\right)$ in $s$, then $s x=s y$ for every $s \in s^{n}=\left\{s_{i} s_{2} \ldots \ldots s_{n} \mid s_{i} \in s\right\}$.

PROOF. Necessity. Let $x, y \in X$ and $\left(s_{i}\right) \quad s$ such that $1 i m s_{i} x=1 i m s_{i} y$. Taking subnet if necessary, we have $\left[11 \mathrm{~m} \pi\left(s_{1}\right)\right](x)=\left[11 \mathrm{~m} \pi\left(s_{1}\right](y)\right.$. Then, by hypothesis, $\zeta(x)=\zeta(y)$ for every $\zeta \in E(S, X)^{n}$. Since $\pi\left(S^{n}\right) \subseteq E(S, X)^{n}$, it follows that $s x=s y$ for every $s \in s^{n}$. Sufficiency. Let $x, y \varepsilon X$ and $\xi=11 \mathrm{~m} \pi\left(s_{k}\right) \varepsilon E(S, X)$ such that $\xi(x)=\xi(y)$. Then by hypothesis, sx = sy for $s \varepsilon S^{n}$. Let $\zeta \in E(S, x) \stackrel{n}{\cdot}$ Then, $\zeta=\zeta_{1}{ }^{\circ} \zeta_{2} \circ \ldots \ldots \ldots \ldots . \zeta_{n}$ where

$\zeta_{j}=\lim _{i j} \pi\left(s i_{j}\right) . \quad$ By induction one can easily show that $\zeta_{1} \circ \zeta_{2} \circ \ldots \ldots \ldots \circ \zeta_{n}(x)$

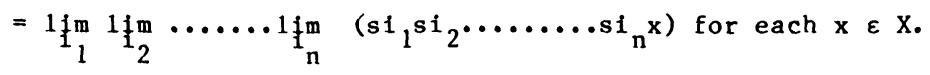

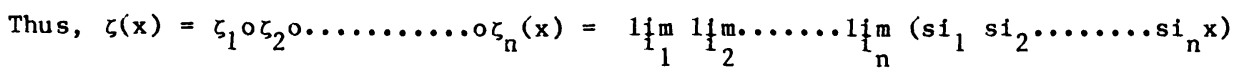

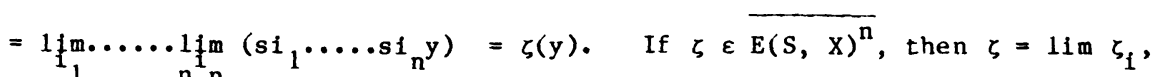
$\zeta_{i} \varepsilon E(S, X)^{n}$, and $\zeta(x)=11 m \zeta_{i}(x)=11 m \zeta_{i}(y)=\zeta(y)$. This completes the proof.

We note that if $S=S^{2}$, then $D^{1}(S)=D^{2}(S)=\ldots \ldots=D^{n}(S)=D^{\infty}(s)$, and that if $s$ has an identity, then $D(S)=D^{n}(S)=D^{\infty}(S)$.

EXAMPLES. i) Trivially all distal functions are $n-$ and $\infty$ distal functions. (ii) Let $S$ be the semigroup of all strictly upper triangular matrices (elements on the diagonal and below are zero) of order $n+2$ with entries from reals. With discrete topology, it is a topological semigroup and $D^{n}(S)=L M C(S)=C(S)$. Defining $g: S+R$ by $g(s)=\left(c_{1}, n+2 v 0\right) \Lambda 2, s=\left(c_{1}, j\right) \varepsilon S$, one verifies that $g$ e $D^{n}(S)$ and $g \in D^{n-1}(S)$. (iii) Let $(\mathrm{N},+)$ be the semigroup of positive integers with discrete topology. Define $f_{n}(t)=1 / t$ if $t<n+1$ and $=0$ if $t>n+1$. Again, it is easily verified that $f_{n} \varepsilon D^{n}(N)$ and $f_{n} \in D^{n-1}(N)$. Later we give an example $f \in D^{\infty}(S)$ but $\varepsilon D^{n}(S)$ for any n.

Using the structure theory of compact right topological semigroups, one may readily prove the following result of $R$. Ellis: (S, X) is distal if and only if $E$ (S, $X$ ) is a group with respect to function composition and with identity, the identity function [4, Proposition 5.3]. We have a more general result corresponding to generalized distal flows.

PROPOSITION 4. A flow (S, $X)$ is n-distal (o-distal) if and only if $\overline{E(S, X)^{n}}\left(n \overline{\left.E(S, X)^{n}\right)}\right.$ is left simple.

PROOF. We first prove the n-case. Necessity. Let $Z=\overline{E(S, X)^{n}}$. It suffices to prove that pe $=p$ for al1 $p \in Z$ and $e \varepsilon E(Z)$. Let $x \varepsilon X$ and $e \varepsilon E(Z)$. Then $e$ is 
also an idempotent of $E(S, X)$, and $e(x)=e(e(x))$. Therefore by definition of $n-$ distal, $p(x)=p(e(x))$ for all $p \varepsilon z$, and hence, $p=$ pe. Sufficiency. Let $x$, $y \varepsilon X$ such that $p(x)=p(y)$ for some $p \in E(S, X)$. Then $p^{n}(x)=p^{n}(y)$ where $\mathrm{p}^{\mathrm{n}} \in \mathrm{E}(\mathrm{S}, \mathrm{X})^{\mathrm{n}}$. As $\mathrm{Z}$ is left simple, $Z=z \mathrm{p}^{\mathrm{n}}$. For any $q \varepsilon Z, q=\mathrm{rp}^{\mathrm{n}}$ where $r \varepsilon z$, and $q(x)=\left(r p^{n}\right)(x)=r\left(p^{n}(x)\right)=r\left(p^{n}(y)\right)=r p^{n}(y)=q(y)$. Hence, the f1ow is n-distal. The proof of the o-case is similar. We omit the necessity part and supply the sufficiency part. Sufficiency. Let $x, y \varepsilon X$ and $p \varepsilon E(S, X)$ such that $p(x)=p(y)$. Then, $p^{n}(x)=p^{n}(y)$ for every $n$. As $p^{n} \varepsilon E(S, x)$, a compact space, there exists a subsequence of $\left(p^{n}\right)$, call it $\left(q_{n}\right)$, such that $q_{n} \rightarrow q_{0}$ in $E(S$, $X)$. It is readily verified that $q_{0} \varepsilon \cap E(S, X)^{n}$ and $q_{0}(x)=q_{0}(y)$. Since $z=n E(S, X)^{n}$ is left simple, $Z=z_{q 0}$. Let $\zeta \varepsilon z$. Then $\zeta=\zeta_{1} q_{0}$ for some $\zeta_{1}$ in $z$. Now,

$\zeta(x)=\zeta_{1}\left(q_{0}(x)\right)=\zeta_{1}\left(q_{0}(y)\right)=\zeta_{1} q_{0}(y)=\zeta(y)$, and this completes the proof.

LEMMA 5. Let $S$ be a semitopological semigroup, (X, $\alpha$ ) the canonical LMC(S)compactification of $S$, and $f \in \operatorname{LMC}(S)$.

i) The following statements are equivalent.
a) $f \in D^{n}(S)$.
b) $T_{u e v} f=T_{u v} f$ for all $u \varepsilon \overline{X^{n}, v} \varepsilon X$, and $e \varepsilon E(X)$
c) $\operatorname{uev}\left(f=u v(f)\right.$ for $a 11 u \varepsilon X^{n+1}, v \varepsilon X$ and $e \varepsilon E(X)$.

11) The following statements are equivalent.
a) $f \in D^{\infty}(S)$
b) $T_{u e v} f=T_{u v} f$ for all $u \varepsilon \cap \overline{x^{n}}, v \underline{\varepsilon} X$, and $e \varepsilon E(X)$.
c) $u e f(f)=u v(f)$ for all $u \varepsilon X .\left(n x^{n}\right), v \varepsilon X$, and $e \varepsilon E(X)$.

PROOF. For $x \in X$, let $T_{x}$ be the left-m-introversion operator determined by $x$. Then, $Z=$ the closure of $R_{S} f$ in the topology of pointwise convergence on C(S) $=\left\{T_{x} f: x \in X\right\}\left[2, \quad\right.$ Lemma 4.19]. Defining $k: X+E(S, Z)$ by $k(x)\left(T_{y} f\right)=T_{x y} f$, one verifies that $k$ is a continuous homomorphism of $X$ onto $E(S, Z)$ satisfying $k o \alpha=\pi$.

i) a) $===\Rightarrow$ b) Let $u \in x^{n}, v \varepsilon X$, and $e \varepsilon E(X)$. Then, $k(u) \varepsilon E(S, z)^{n}$, and $k(e)$ is an idempotent of $E(S, Z)^{n}$. As $E(S, Z)^{n}$ is left simple (hypothesis), $k(u) k(e)=k(u)$, i.e., $k(u e)=k(u)$. In particular, $k(u e)\left(T_{v} f\right)=k(u)\left(T_{v} f\right)$ where $T_{v} f \varepsilon z$, i.e., $T_{u e v} f$ $=T_{u v} f$. Since $X$ is right topological with w topology, it follows that $T_{u e v} f=T_{u v} f$ for all $u \in \overline{x^{n}}, \quad v \varepsilon X$, and $e \varepsilon E(X)$.

b) $===\Rightarrow$ c) Let $u \in X^{n+1}, v \varepsilon X$, and $e \varepsilon E(X)$. Then, $u=u_{1} u_{2}$, where $u_{1} \varepsilon X$, $u_{2} \varepsilon x^{n}$, and $u e v(f)=u_{1} u_{2} e v(f)=u_{1}\left(T_{u_{2}}\right.$ evf $)=u_{1}\left(T_{u_{2}}\right.$ ff $)=u_{1} u_{2} v(f)=u v(f)$. Thus, uev $(f)=u v(f)$. It is easily verified that uev(f) $=u v(f)$ for $u \varepsilon x^{\overline{n+1}}$.

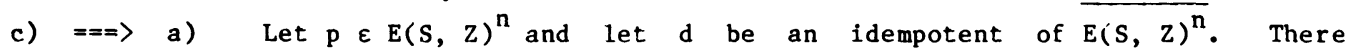
exists $u \in X^{n}$, e $\varepsilon E(X)$ such that $k(u)=p$, and $k(e)=d$. Such a choice of $e$ is possible as $k^{-1}(d)$ is a compact subsemigroup of $X$. Let $v \varepsilon X$. For any $w \varepsilon X$, $w\left(T_{\text {uev }} f\right)=\operatorname{wuev}(f)=\operatorname{wuv}(f)$ (hypothesis) $=w\left(T_{u v} f\right)$. Therefore, $T_{u e v} f=T_{u v} f$. Now, $k(u e)\left(T_{v} f\right)=T_{u e v} f=T_{u v} f=k(u)\left(T_{v} f\right)$, which implies that $k(u e)=k(u)$. Thus, $\mathrm{pd}=\mathrm{k}(\mathrm{ue})=\mathrm{k}(\mathrm{u})=\mathrm{p} \overline{\mathrm{pd}}=\mathrm{k}(\mathrm{ue})=\mathrm{k}(\mathrm{u})=\mathrm{p}$. As $\mathrm{E}(\mathrm{S}, \mathrm{z})^{\mathrm{n}}$ is right topological, it follows that $p d=p$ for all

$p \varepsilon \overline{E(S, Z)^{n}}$ proving that $\overline{E(S, Z)^{n}}$ is left simple. Consequently, the flow

$(S, Z, \pi)$ is $n$-distal, and thus, $f \in D^{n}(S)$. i1) The proofs of a) $\Rightarrow b$ ) and b) $\Rightarrow \Rightarrow$

c) in i) are easily modified to prove the corresponding results in ii). Let us prove 
the case $c) \Rightarrow a)$. It suffices to show that $\cap E(S, Z)^{n}$ is left simple. Let $\mathrm{p} \varepsilon \cap \overline{E(S, Z)^{\mathrm{n}}}$ and let $\mathrm{d}$ be an idempotent of $n \overline{E(S, Z)^{\mathrm{n}}}$. There exists an $e \varepsilon E(X)$ such that $k(e)=d$. We prove the existence of an $u$ in $n x^{n}$ such that $k(u)$ $=p$. Let $n$ be fixed and $p \in \bar{E}(S, Z)^{n}$. Then, $p=11 m p_{i}$ for some $\left(p_{i}\right) \subseteq E(S, Z)^{n}$. For each $p_{1}$, there exists $x_{i} \varepsilon x^{n}$ such that $k\left(x_{1}\right)=p_{1}$. Now $\left(x_{1}\right)\left(\subseteq x^{n}\right)$ has a convergent subnet $\left(x^{j}\right)$ converging to an element, call it $x_{n}$, in $x^{n}$. $p_{j}=k\left(x^{j}\right) \frac{\text { which }}{n}$ converges to $k\left(x_{n}\right)$ and hence, $p=k\left(x_{n}\right)$. We now have a sequence $\left(x_{n}\right) \subseteq x,\left(x_{n} \varepsilon x^{n}\right)$, having a convergent subsequence $\left(x_{n}^{\prime}\right)$ such that $x_{n}^{\prime}+u$ in $x$. One readily verifies that $u \varepsilon \cap \overline{x^{n}}$ and that $k(u)=p$. We omit the rest of the proof which is similar to the proof of c) $=\Rightarrow$ a) in 1 ).

THEOREM 6. a) $D^{\mathrm{n}}(\mathrm{S})$ and $D(S)$ are admissible subalgebras of $C(S)$. b) The $D^{n}(S)-\left(D^{\infty}(S)-\right)$ compactification $(Y, \beta)$ of $S$ is maximal with respect to the property that

(1) uev $=u v$ for $u \in \overline{Y^{n+1}}\left(u \in Y, n \overline{Y^{n}}\right), v \varepsilon Y$, and $e \varepsilon E(Y)$

PROOF. a) Let $(X, \alpha)$ denote the canonical LMC-compactificaion of $S$. That $D^{n}(S)$ is a linear subspace of $C(S)$ is immediate from Lemma 5 (i). It is easily verified that $D^{n}(S)$ is norm closed. Let $f \in D^{n}(S), u \varepsilon X^{n+1}, v \varepsilon X, e \varepsilon E(X)$, and $s \varepsilon S$. Then, $\operatorname{uev}\left(L_{s} f\right)=\alpha(s) \operatorname{uev}(f)=\alpha(s) u v(f)=u v\left(L_{s} f\right)$. Hence, $D^{n}(S)$ is left translation invariant. In a similar manner, one verifies that $D^{n}(S)$ is right translation invariant. The fact that $X$ is the set of all multiplicative means proves that $D^{n}(S)$ is an algebra. As uev(1) $=u v(1), D^{n}(S)$ contains all the constant functions. Let $w^{\prime} \varepsilon M M\left(D^{n}(S)\right)$ and $f \in D^{n}(S)$. Let $\theta: X+M M\left(D^{n}(S)\right)$ be the restriction map. There exists a $w$ in $X$ such that $\theta(w)=w^{\prime} \cdot T_{w} f=T_{w_{n}}, f$, and $\operatorname{uev}\left(T_{w^{\prime}}, f\right)=\operatorname{uev}\left(T_{w} f\right)=\operatorname{uevw}(f)=$ $u_{v w}(f)=u v\left(T_{w} f\right)=u v\left(T_{w}, f\right)$. Thus, $T_{w}, f \in D^{n}(S)$ which proves that $D^{n}(S)$ is left $m$ introverted. Thus, $D^{n}(S)$ is an admissible algebra of $C(S)$. The proof that $D^{\infty}(S)$ is an admissible algebra is similar. b) We give the proof for the $\infty$-case, and omit the proof for the $n$-case. Let $(X, \alpha)$ denote the canonical LMC-compactification of $s$. Let $\theta: X+Y$ denote the restriction mapping. The $\theta$ is a continuous homomorphism of $X$ onto $Y$ such that $\theta 0 \alpha=\beta$. First, we prove that $Y$ has the property ( 1 ). Let

$u \varepsilon Y . \cap \overline{Y^{n}}, v \varepsilon Y$, and $e \varepsilon E(Y) . u=u_{1} u_{2}$ where $u_{1} \varepsilon Y$ and $u_{2} \varepsilon \cap Y^{n}$. There exist $\mathrm{x}_{1}, \mathrm{y} \varepsilon \mathrm{X}, \mathrm{d} \varepsilon \mathrm{E}(\mathrm{X})$, and $\mathrm{x}_{2} \varepsilon \cap \overline{\mathrm{X}^{\mathrm{n}}}$ such that $\theta\left(\mathrm{x}_{1}\right)=\mathrm{u}_{i}(i=1,2), \theta(\mathrm{y})=\mathrm{v}$, and $\theta(d)=$ e. Therefore, for any $f \varepsilon D^{\infty}(S), \operatorname{uev}(f)=\theta(x d y)(f)=x \operatorname{xy}(f)=x y(f)$ $[$ Lemma $5 \mathrm{ii})]=\theta(\mathrm{xy})(\mathrm{f})=\mathrm{uv}(\mathrm{f})$. Hence, uev $=\mathrm{uv}$, and thus, $Y$ has the property (1). To prove that $(Y, B)$ is maximal with respect to this property, it remains to show that $\beta_{0}^{*} C\left(Y_{0}\right) \subseteq D^{\infty}(S)$ for any right topological compactification $\left(Y_{0}\right.$, $\left.\beta_{0}\right)$ of $S$ having property (1), where $\beta_{0}^{*}: C\left(Y_{0}\right)+C(S)$ is the adjoint of $B_{0}$. It is shown that $\beta_{0}^{*} C\left(Y_{0}\right) \subseteq \operatorname{LMC}(S) \quad[5$, page 385]. Therefore, there exists a continuous homomorphism $\delta: X+Y_{0}$ such that $\beta_{0}=\delta o \alpha$ Let $g \varepsilon C\left(Y_{0}\right)$ and $\beta_{0}^{*} g=f$. Now, $\alpha(s)(f)=\alpha(s)\left(\beta_{0}^{*} g\right)=\beta_{0}^{*} g(s)=g\left(\beta_{0}(s)\right)=g(\delta(\alpha(s)))$. $\quad$ By taking 1imits, $x(f)=g\left(\delta(x)\right.$ ) for $x \varepsilon X$. Let $u \varepsilon X . \cap \overline{X^{n}}, v \varepsilon X$, and $e \varepsilon E(X)$. Clearly, $\delta(\mathrm{u}) \varepsilon \mathrm{Y}_{0^{\circ}} \cdot \overline{\mathrm{Y}} \mathrm{Y}_{0}^{\mathrm{n}}, \delta(\mathrm{v}) \varepsilon \mathrm{Y}_{0}$, and $\delta(\mathrm{e})$ is an idempotent of $\mathrm{Y}_{0}$. Then uev(f) $=g(\delta($ uev $))=g(\delta(u) \delta(e) \delta(v))=g\left(\delta(u) \delta(v)\right.$ ) (since $Y_{0}$ has property

$=g(\delta(u v))=u v(f)$. Thus, $f \varepsilon D^{\infty}(S)$ and this completes the proof. 
3. INVERSE LIMITS AND $D^{\mathrm{n}}(\mathrm{S})$-COMPACTIFLCATLONS.

In this section, we prove that $U D^{n}(S)$ is an admissible subalgebra of $C(S)$, and its compactification $(X, \alpha)$ is the inverse 1 imit space of the spectrun $\left\{x_{n}, \pi_{n m}\right\}$ where

$\left(X_{n}, \alpha_{n}\right)$ is the $D^{n}(S)$-compactification of $S$ and $\pi_{n m}: X_{n}+X_{m} \quad(n>m)$ is the restriction map. For definition and terminologies in inverse limits, we shall follow Dugundji [3]. Let $I$ be a preordered set and $\left\{x_{\xi}\right\}_{\xi \in I}$ be a family of topological spaces. For $\xi>\zeta$, assume there is given $\pi_{\xi \zeta}: x_{\xi}+x_{\zeta}$ a continuous map such that whenever

$\xi>\zeta>n, \quad \pi_{\xi \eta}=\pi_{\zeta \eta} \circ \pi_{\xi \zeta^{\circ}} \quad$ Then the family $\left\{x_{\xi}: \pi_{\xi \zeta}\right\}$ is called an inverse spectrum over I. The subspace $\left\{x \in \pi_{\xi}: \zeta \leqslant \xi \Rightarrow P_{\zeta}(x)=\Pi_{\xi \zeta} \circ P_{\xi}(x)\right\}$, where $\mathrm{P}_{\xi}: \pi \mathrm{x}_{\xi}+\mathrm{x}_{\xi}$ is the projection map, is called the inverse limit spectrum of the spectrum and is denoted by $x_{\infty}$.

THEOREM 7. Let $X$ be a compact topological space, and $\left\{x_{\xi}\right\}_{\xi} \varepsilon I$, indexed by a directed set $I$, be a family of topological spaces. Assume there are given $\Pi_{\xi}: X+x_{\xi}$ for every $\xi$ and for $\xi>\zeta, \pi_{\xi \zeta}: x_{\xi}+x_{\zeta}$ surfective, continuous, and consistent maps (i.e., for $\left.\xi>\zeta, \pi_{\xi \zeta}{ }^{0} \pi_{\xi}=\pi_{\zeta}\right)$ such that for any two distinct points $x_{1}, x_{2} \varepsilon x$, there exists $\xi \in I$ such that $\pi_{\xi}\left(x_{1}\right) \neq \pi_{\xi}\left(x_{2}\right)$. Then $X$ is homeomorphic to the inverse 1 imit space of the spectrum $\left\{x_{\xi}: \pi_{\xi \zeta}\right\}$.

PROOF. The hypotheses imply that for $\xi>\zeta>n, \pi_{\xi \eta}=\pi_{\zeta \eta^{\circ}} \pi_{\xi \zeta^{\circ}}$ Therefore $\left\{x_{\xi}: \pi_{\xi \zeta}\right\}$ is an inverse spectrum over I. Set

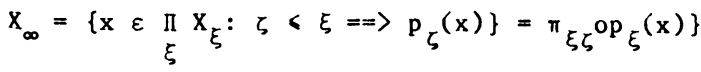

$$
\begin{aligned}
& \left\{x \in \pi_{\xi} x_{\xi}: \zeta<\xi \Rightarrow x_{\zeta}=\pi_{\xi \zeta}\left(x_{\eta}\right) \text { where } p_{\eta}(x)=x_{\eta}\right\} \text {. }
\end{aligned}
$$

Define $\theta: X \rightarrow x_{\infty}$ as $\theta(x)=\left(\pi_{i}(x)\right)_{i \in I}$. We complete the proof by showing that $\theta$ is a homeomorphism. If $\zeta<\xi$, then $p_{\zeta}(\theta(x))=\pi_{\zeta}(x)=\pi_{\xi \zeta}$ o $\pi_{\xi}(x)$ (by consistency of maps $)=\pi_{\xi \zeta} \zeta_{\xi}(\theta(x))$. Hence, $\theta(x) \varepsilon x_{\infty}$. If $x_{1}, x_{2}$ are two distinct points of $x$, then by hypothesis, there exists $\xi \in I$ such that $\pi_{\xi}\left(x_{1}\right) \neq \pi_{\xi}\left(x_{2}\right)$. This implies that $\theta\left(x_{1}\right) \neq \theta\left(x_{2}\right)$ and hence, $\theta$ is injective. Let $y \varepsilon x_{\infty}$. For $\zeta<\xi$, we prove that $\left.\pi_{\xi}^{-1}\left(p_{\xi}\right)\right) \subseteq \pi_{\zeta}^{-1}\left(p_{\zeta}(y)\right)$. Let $z \in \pi_{\xi}^{-1}\left(p_{\xi}(y)\right)$. Then, $\pi_{\xi}(z)=p_{\xi}(y)$ and $\pi_{\zeta}(z)=\pi_{\xi \zeta}{ }^{0} \pi_{\xi}(z)$ (consistency of maps) $=\pi_{\xi \zeta}{ }^{\circ} p_{\xi}(y)=p_{\zeta}(y)$ (since y $\varepsilon x_{\infty}$ ). Therefore, $z \in \pi_{\zeta}^{-1}\left(p_{\zeta}(y)\right)$. Thus we see that, if $t, t_{1}, t_{2}, \ldots \ldots \ldots t_{n}$, are arbitrary members of $I$ such that $t>t_{i}(1<i<n)$, then

$$
\pi_{t}^{-1}(y t) \subseteq \pi_{t}^{-1}(y) \neq \phi
$$

As $\pi_{t}$ is continuous for each $t$ and $\left\{y_{t}\right\}$ is closed, $\left\{\pi_{t}^{-1}\left(y_{t}\right): t \varepsilon I\right\}$ is a class of closed sets in $X$, a compact space, with every finite intersection being nonempty (by (2)). Therefore, $t \varepsilon_{\varepsilon I}^{n} \pi_{t}^{-1}\left(y_{t}\right) \neq \phi$. For any $x \in{ }_{t \in I \pi_{t}^{-1}}^{n}\left(y_{t}\right), \theta(x)=y$, and hence $\theta$ is surjective. Clearly $\theta$ is continuous. Since $X$ is compact, $\theta$ is a homeomorphism.

THEOREM 8. Let $\left\{F_{\xi}\right\}_{\xi \varepsilon I}$ indexed by a directed set $I$, be a family of admissible subalgebras of $C(S)$ such that $F_{n} F_{\xi}(n<\xi)$ and $\left(X_{\xi}, a_{\xi}\right)$ is the $F_{\xi}$-compactification of s. Then $F=\overline{U F}_{\zeta}$ is admissible and the F-compactification $(X, \alpha)$ is the inverse 1 imit space of the $\xi^{\text {ppectrum }}\left\{X_{\xi}: \pi_{\xi \eta}\right\}$ where $\pi_{\xi \eta}: X_{\xi}+X_{n}(\eta<\xi)$ is the restriction map $\left(\pi_{\xi \eta}(\mu)=\mu \mid F_{\eta}\right)$. 
PROOF. Since $I$ is directed, it is easily seen that. $F$ is admissible. Define $\pi_{\nu}: X+X_{\nu}$ as the restriction map. Then, in view of theorem 7 , it suffices to prove that for any two distinct points $x_{1}, x_{2} \varepsilon X$ there exists $n \varepsilon$ I such that

$\pi_{n}\left(x_{1}\right) \neq \pi_{n}\left(x_{2}\right)$. The fact that $x_{1}, x_{2} \varepsilon x$ and $x_{1} \neq x_{2}$ implies that there exists an $f \varepsilon F$ such that $\pi_{\mu}\left(x_{1}\right) \neq \pi_{\eta}\left(x_{2}\right)$. The fact that $x_{1}, x_{2} \varepsilon x$ and $x_{1} \neq x_{2}$ implies that there exists an $f \in F$ such that $x_{1}(f) \neq x_{2}(f)$. By continuity of $x_{1}$ and $x_{2}$, there exist $\eta \in I$ and $g \in F_{\eta}$ such that $\pi_{\eta}\left(x_{1}\right)(g)=x_{1}(g) \neq x_{2}(g)=\pi_{\eta}\left(x_{2}\right)(g)$. Hence, $\pi_{n}\left(x_{1}\right) \neq \pi_{n}\left(x_{2}\right)$ and that completes the proof. The following theorem is an immediate cornllary to theorem 8 .

THEOREM 9. $U \mathrm{D}^{\mathrm{n}}(\mathrm{S})$ is an admissible subalgebra of $C(S)$ and its compactification $(X, \alpha)$ is the inverse limit space of the spectrum $\left\{x_{n}: \pi_{n m}\right\}$ where $\left(X_{n}, \alpha_{n}\right)$ is the $D^{n}(S)$-compactification of $S$ and $\pi_{n m}: X+X_{m}(n>m)$ is the restriction map. It is clear that $D^{n}(S) \subseteq D^{\infty}(S)$. Now, we give an example of a function $f \in D^{\infty}(S)$ but

$D\left(G^{n}\right)$ for any $n$. Let $f_{n}$ be defined as in Example i1i. Defining $f(t)$ as $f(t)=$ $1 / t, t \varepsilon N$, we see that $f_{n} \rightarrow f$ (norm). Thus, $f \varepsilon \cup D^{n}(N) \subseteq D^{\infty}(N)$. Clearly,

$f \notin D(N)$. We remark that at this point we do not know whether the containment in $U D^{n}(S) \subseteq D^{\infty}(S)$ is proper.

\section{FIXED POINT THEOREM.}

Let $F$ be a norm closed, conjugate closed, left (right) translation invariant subspace of $C(S)$ containing constants. Then a mean $\mu$ on $F$ is called left (right) invariant if for each $f \varepsilon F, \quad s \varepsilon S, \mu\left(L_{s} f\right)=\mu(f)\left[\mu\left(R_{s} f\right)=\mu(f)\right]$. A left (right) translation invariant subspace $F$ of $C(S)$ is said to be left (right) amenable if there is a left (right) invariant mean on $F$, and amenable if $F$ is translation invariant and both left and right amenable. L. N. Argabright [1] has proved that $F$ is left amenable if and only if every affine flow $(S, X, \pi)$ such that $\left\{x \in X: U_{X} A(X) \subseteq F\right\} \neq \phi$ has a fixed point, where $A(X)$ denotes the Banach space of all continuous complex valued affine functions on $X$, and $U_{X}: C(X)+C(S)$ is defined as $U_{x} h(s)=h(s x), s \varepsilon s, h \varepsilon C(X)$, and $x \in X$. We make use of this result to prove the following fixed point theorem. Let us prepare a lemma for proving the theorem. Defining $\pi: S+M(F)^{M(F)}$ as $\pi(s)(x)=L_{s}^{*} x$, where $L_{s}^{*}$ denotes the adjoint of $L_{s}: F \rightarrow F$, one verifies that, relative to the action $(s, x) \rightarrow L_{s}^{*} x,(S, M(F), \pi)$ is affine flow. If in addition, $F$ is an algebra, then $M(F)$ is a closed invariant subspace of $M(F)$, and relative to the restricted action, $(S, M M(F), \pi)$ is a flow. These actions of $S$ are called the natural actions of $S$ on $M(F)(M M(F))$. Let $\left(Z=M M\left(D^{n}(S), B\right)\right.$ denote the canonical $D^{n}(S)-$ compactification of $S$. Then relative to the natural action, $(S, Z, \pi)$ is a flow.

LEMMA 10. The flow $(S, Z, \pi)$ is $(n+1)$-distal.

PROOF. Let $z_{1} z_{2} \in Z$ and $\left(s_{1}\right) \subseteq S$ such that $1 \mathrm{im} s_{1} z_{1}=1 \mathrm{~m} s_{1} z_{2}$. I.e., $\lim B\left(s_{i}\right) z_{1}=11 \mathrm{~m} B\left(s_{i}\right) z_{2}$. Taking subnet if necessary, $z_{0} z_{1}=z_{0} z_{2}$ where $z_{0}=\lim \beta\left(s_{1}\right) \varepsilon z$. Hence $y z_{0} z_{1}=y z_{0} z_{2}$ for every $y \varepsilon Z$, from which it follows that $\mathrm{zz}_{1}=\mathrm{zz}_{2}$ for each $z \varepsilon \mathrm{zz}_{0} 0^{\circ}$ Now, $\mathrm{zz}_{0}$, being a left ideal in $\mathrm{z}$, a compact right topological semigroup, has an idempotent element e. Thus $e_{1}=e z_{2}$. For s $\varepsilon s^{n+1}, s z_{1}=\beta(s) z_{1}=\beta(s) e z_{1} \quad\left(\right.$ Theorem 6) $=\beta(s)$ ez $z_{2}=s z_{2}$. Therefore, $(S, Z, \pi)$ is $(n+1)$ - distal (Prop. 3).

THEOREM 11. (Fixed Point Theorem) $\mathrm{D}^{\mathrm{n}}(\mathrm{S})$ is left amenable if and only if every affine flow $(S, Y, \pi$ ) containng a closed invariant subspace $Z$ such that $(S, Z, \pi)$ is $(n+1)$-distal has a fixed point. 
PROOF. Let $D^{n}(S)$ be left amenable. Suppose that. $(S, Y, \pi)$ is an affine flow containing a closed invariant subspace $Z$ such that $(S, Z, \pi)$ is $(n+1)$-distal. We show that $\left\{x \in Y: U A(Y) \subseteq D^{n}(S)\right\} \neq \phi$. Let $x \in Z$ and $h \in A(Y)$. It is easily seen that ${ }_{S_{S}} U_{x} h=U_{S x}{ }^{h}$. Let $\left(U_{S i} x^{h}\right)$ be a net in $U_{S x} h$. The net $\left(s_{i} x\right)$ in $Z$ has a convergent subnet $\left(s_{j} x\right)$ converging to some point $x_{0}$ in $Y$. As $\pi(s): Y+Y$ is continuous, $s s_{j} x+s_{h}$ for every $s \varepsilon s$. Hence, $h\left(s s_{j} x\right)+h\left(s x_{0}\right)$ for every $s \varepsilon s$. It follows that $U_{s j} x^{h} \rightarrow U_{x_{0}} h$ (pointwise). This proves that $U_{S x}{ }^{h}=R_{S} U_{x} h$ is relatively compact in the pointwise topology, and thus, $U_{x} h \varepsilon L M C(S)$. Let $(X, \alpha)$ denote the canonical LMCcompactification of $S$. As $(E(S, Z), \pi)$ is a right topological compactification of $S$, by the universal mapping property of $(X, \alpha)$, there exists $\phi: X+E(S, Z)$, a continuous homomorphism, such that $\phi \circ \alpha=\pi$. Then $\alpha(s)\left(U_{x} h\right)=h(s x)=h(\pi(s)(x))=$

$h\{(\phi \circ \alpha)(s)(x)\}$. Taking limits ( $\alpha$ has dense range in $x$, and $h, \phi$ are continuous), we get $u\left(U_{x} h\right)=h(\phi(u)(x))$ for each $u \varepsilon x$. Let $u \varepsilon x^{n+1}, v \varepsilon x$, and $e \varepsilon E(x)$. Then $\phi(u) \varepsilon E(S, Z)^{n+l}, \phi(v) \varepsilon E(S, Z)$, and $\phi(e)$ is an idempotent in $E(S, Z)$. As $E(S, Z)^{n+1}$ is left simple $\phi(u) \phi(e)=\phi(u)$. Hence, uev $\left(U_{x} h\right)=h(\phi(u e v)(x))=$

$h(\phi(u) \phi(e) \phi(v)(x))=h(\phi(u) \phi(v)(x))=h(\phi(u v)(x))=u v\left(u_{x} h\right)$. $\quad$ As $x \quad$ is right topological, it follows that $\operatorname{uev}\left(U_{x} h\right)=u v\left(U_{x} h\right)$ for any $u \varepsilon \overline{x^{n+1}}$. Thus,

$U_{x} h \in D^{n}(S)$. This proves the necessary part. For Sufficiency, let $Y=M\left(D^{n}(S)\right)$ and define $\pi(s): Y+Y$ as $\pi(s)(x)=L_{s}^{*} x, s \in S, \quad X \in Y$. Then (S, Y, $\left.\pi\right)$ is an affine flow. Let $(Z, \beta)$ denote the canonical $D^{n}(S)$ - compactification of $S$. Then the flow $(S, Z, \pi)$ is $(n+1)$-distal (Lemma 10). So by hypothesis, $(S, Y, \pi)$ has a fixed point $y_{0}$ such that $y_{0}=s y_{0} \quad\left(=L_{s}^{*} y_{0}\right)$ for every $s \in s$. Hence

$y_{0}(f)=L_{s}^{*} y_{0}(f)=y_{0}\left(L_{s} f\right)$ for every $s \in S$ and for every $f \in D^{n}(S)$. Therefore, $y_{0}$ is a left invariant mean on $D^{n}(S)$.

\section{REFERENCES}

1. ARGABRIGHT, L. Invariant Means and Fixed Points, A sequel to Mitchell's paper, Trans. Amer. Math. Soc. 130 (1968), 127-130.

2. BERGLUND, J., JUNGHEN, H. and MILNES, P. Compact Right Topologica1 Semigroups and Generalizations of Almost Periodicity, Lecture Notes in Mathematis 663, Springer-Verlag, New York, 1978.

3. DUGUNDJI, J. Topology, Prnetice-Ha11 International, Inc., London, 1966.

4. ELLIS, R. Lectures on Topologica1 Dynamics, Benjamin, New York, 1969.

5. JUNGHENN, H. Distal Compactifications of Semigroups, Trans. Amer. Math. Soc. $274(1982), 379-397$.

6. MILNES, P. Compactifications of Semitopological Semigroups, Austra1. Math. Soc. $15(1973), 488-503$. 


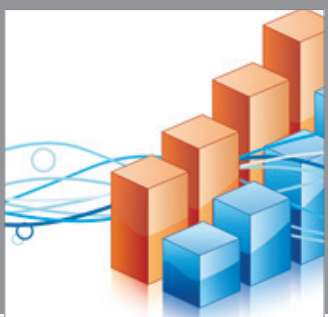

Advances in

Operations Research

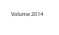

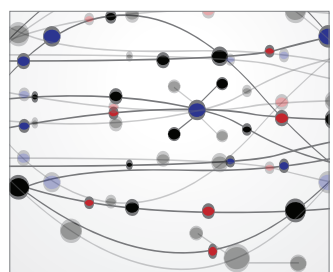

\section{The Scientific} World Journal
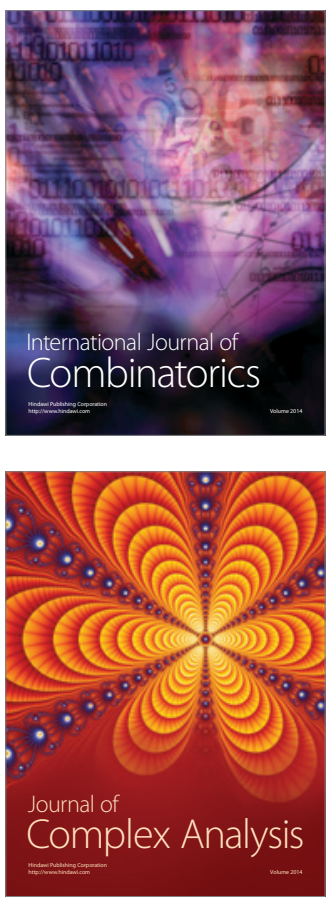

International Journal of

Mathematics and

Mathematical

Sciences
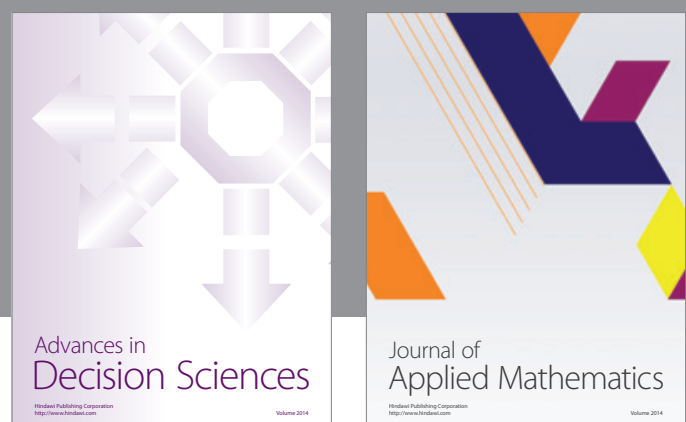

Journal of

Applied Mathematics
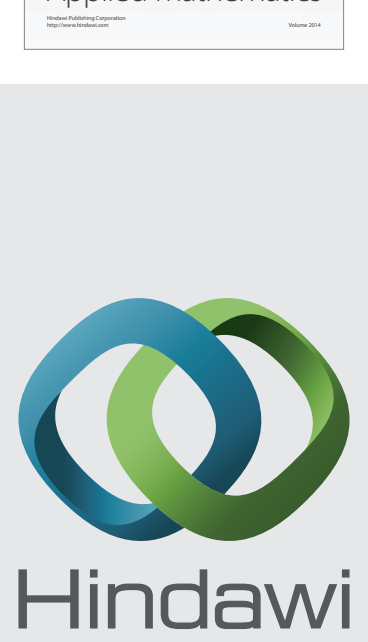

Submit your manuscripts at http://www.hindawi.com
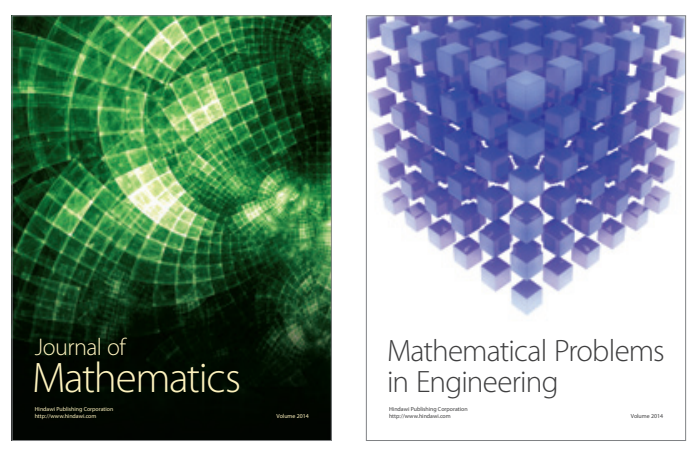

Mathematical Problems in Engineering
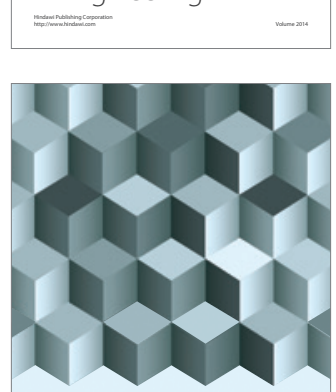

Journal of

Function Spaces
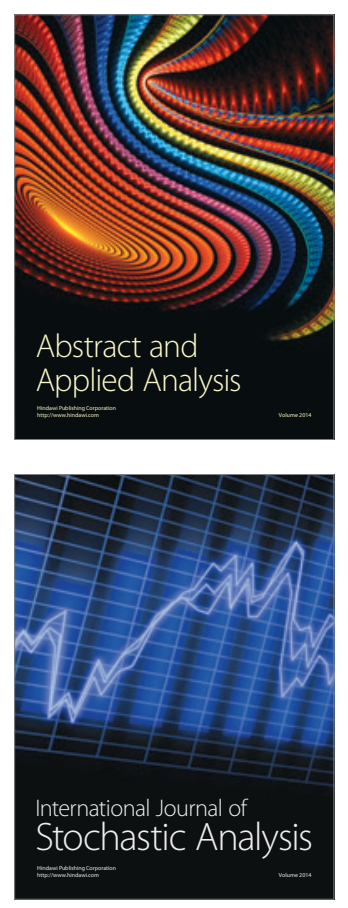

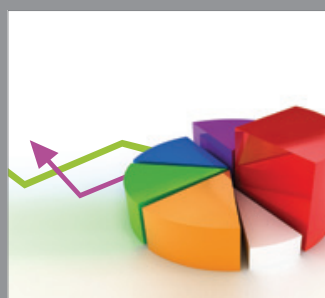

ournal of

Probability and Statistics

Promensencen
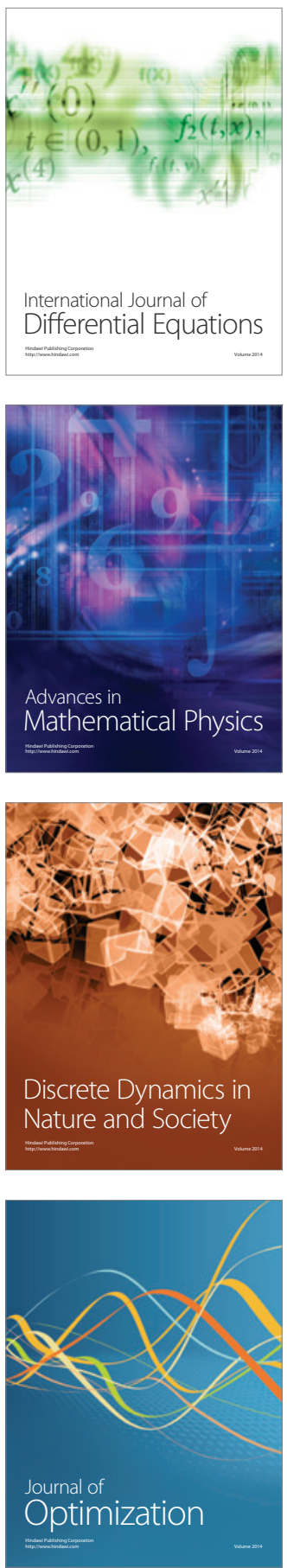